\title{
Minimum Number of Subsets to Distinguish Individual Elements
}

\author{
P. R. Meyers \\ Institute for Basic Standards, National Bureau of Standards, Washington, D.C. 20234
}

(December 9, 1966)

\begin{abstract}
Given a set $S$ of cardinality $m$, we determine the minimum cardinality $f(m)$ for a family $F$ of subsets of $S$ such that each $s \epsilon S$ can be expressed as the intersection of some subfamily of $F$. The problem is solved in the following inverse form. For a given number $n$ of subsets of $S$, find $g(n)$ : the maximum number of elements of $S$ which can be written as the intersection of some of these subsets. We show that $g(n)$ is the largest binomial coefficient for combinations of $n$ things.
\end{abstract}

Key Words: Classification design, combinatorics, set theory.

\section{Introduction}

Let $S$ be a finite set of given cardinality $|S|=m$. An element $s \epsilon S$ will be said to be distinguished by a family $\mathscr{F}$ of subsets of $S$, if $\{s\}$ is the intersection of some subfamily of $\mathscr{F}$. In this note we solve the following combinatorial problem (conveyed by K. E. Kloss): What is the minimum possible cardinality $f(m)$ for a family which distinguishes all elements of $S$ ? (Trivially $f(m) \leqslant m$, since

$$
\mathscr{F}=\{\{s\}: s \epsilon S\}
$$

distinguishes all elements.)

The question may sound like another one which arises fairly naturally in a context of classification design or information retrieval: How many "categories" (subsets) must be established so that any item (element) in a collection can be uniquely specified by listing those categories under which it falls? The categories which uniquely specify some item may be a subcollection of those which specify another item, while any family of subsets with a one element intersection cannot be part of a larger family with a different nonempty intersection.

It will be more convenient to work with the following inverse form of the problem: to determine $g(n)$, the maximum cardinality of a set $S$ of elements which are distinguished by some family $\mathscr{F}=\left\{F_{1}, \ldots, F_{n}\right\}$. The inversion is made precise by removing from each $F_{i}$ those elements of $S$ which are not distinguished by $\mathscr{F}$. (For this question $n$ is fixed, but not $\mathscr{F}$.) It will be shown below that

$$
g(n)=\left(\begin{array}{l}
n \\
[n / 2]),
\end{array}\right.
$$

where $(\cdot)$ is the binomial coefficient and $[n / 2]$ is the largest integer not greater than $n / 2$. This yields an implicit solution to the original problem, since

$$
f(m)=\min \{n: g(n) \geqslant m\},
$$

follows when we observe that

$$
m \leqslant g(n) \text { iff } f(m) \leqslant n .
$$

\section{Proof}

Let $h(n)$ be the binomial coefficient on the right-hand side of (1). We first show that $h(n) \leqslant g(n)$. For this purpose, let $A$ be a set with cardinality $|A|=n$ and let

$$
S^{*}=\left\{s_{1}, \ldots, s_{h(n)}\right\}
$$

be the collection of all subsets of $A$ which have cardinality $[n / 2]$. For $1 \leqslant i \leqslant n$, let

$$
H_{i}=\left\{s \epsilon S^{*}: i \epsilon s\right\},
$$

and put $\mathscr{H}=\left\{H_{1}, \ldots, H_{n}\right\}$. The possibility

$$
r \in \cap\left\{H_{i}: s \in H_{i}\right\},\left(r \in S^{*}-\{s\}\right),
$$

is ruled out because $r$ cannot be a subset of $s$, so that some $i \epsilon A$ must satisfy $i \epsilon s-r$ and thus $s \epsilon H_{i}, r \epsilon S^{*}-H_{i}$. It follows that

$$
\{s\}=\cap\left\{H_{i}: s \in H_{i}\right\},
$$

i.e., each element of $S^{*}$ is distinguished by $\mathscr{H}$. This implies $h(n) \leqslant g(n)$.

The proof of (1) will be completed by showing that $g(n) \leqslant h(n)$. Let $\mathscr{F}=\left\{F_{1}, \ldots, F_{n}\right\}$ be a family of 
finite sets with union $S$. For each $s \epsilon S$, let

$$
F^{(s)}=\left\{F_{i}: s \in F_{i}\right\}, F_{s}=\cap\left\{F_{i}: s \in F_{i}\right\}, T=\left\{s \in S: F_{s}=\{s\}\right\} .
$$

Then $T$ consists of those elements of $S$ which are distinguished by $\mathscr{F}$ so that $|T| \leqslant h(n)$ is what must be proved.

A collection of sets will be called independent if no set-inclusions hold between any pair of members.

For example, the collection $\left\{F^{(s)}: s \in T\right\}$ is an independent collection of subfamilies of an $n$ member family. Since this collection has $|T|$ members, it suffices to show that any independent family of subsets of an $n$ element set has at most $h(n)$ members. This can be shown using the well-known SDR theorem but we find it as easy to employ an elementary argument.

For an $n$ element set $A$, let $S_{i}$ denote the family of subsets of $A$ which have cardinality $i, 0 \leqslant i \leqslant n$. Each $S_{i}$, and in particular $S_{[n / 2]}=S^{*}$, is an independent family. If $\{n / 2\}$ is the smallest integer not less than $n / 2$, then

$$
\left|S_{[n / 2]}\right|=\left|S_{\{n / 2\}}\right|=h(n) .
$$

We shall show that any other independent family $P$ of subsets of $A$ can be mapped $1-1$ into $S_{[n / 2]}$ and thus conclude that

$$
|P| \leqslant\left|S_{[n / 2]}\right| \text {. }
$$

Suppose some member of $P$ has cardinality less than $\{n / 2\}$. Let $P_{j}$ be the family of members of $P$ which have smallest cardinality, say $j$. Let $M$ be the family of members of $S_{j+1}$ which contain a mernber of $P_{j}$. Since $P$ is independent, $P^{\prime}=M \cup\left(P-P_{j}\right)$ is also independent, and $P \cap M=\phi$. We will show below that

$$
j<\left\{\frac{n}{2}\right\} \text { implies }\left|P_{j}\right| \leqslant|M|,
$$

and so $|P| \leqslant\left|P^{\prime}\right|$.
Then by induction on the minimum cardinality of any member of $P, P^{\prime}$, etc., we obtain an independent family $Q$ such that $|P| \leqslant|Q|, Q \cap S_{i}=\phi$ for $0 \leqslant i<\left\{\frac{n}{2}\right\}$, and $Q \cap S_{i}=P \cap S_{i}$ for $\{n / 2\}<i \leqslant n$.

The structure of the family of all subsets of $A$ is the same relative to the relationships "is a subset of" and "is a superset of." Hence a "mirror-image" of the preceding construction will produce from $Q$ an independent family $R$ such that $|Q| \leqslant|R|, R \cap S_{i}=\phi$ for $n \geqslant i>[n / 2]$, and such that

$$
R \cap S_{i}=Q \cap S_{i}=\phi \text { for }[n / 2]>i \geqslant 0 .
$$

In this fashion we arrive at the result $|P| \leqslant|R|$ and $R \subset S_{[n / 2]}$.

It only remains to show (6).

Let $K$ be the number of distinct pairs $(p, m)$ where $p \epsilon P_{j}, m \epsilon M$, and $\mathrm{p} \subset m$. We have

$$
K=(n-j)\left|P_{j}\right|
$$

since any $p \epsilon P_{j}$ can be extended in exactly $(n-j)$ ways to an $m \in M$. Also however,

$$
K \leqslant(j+1)|M|
$$

since any $m \in M$ contains $j+1$ subsets of cardinality $j$ and thus contains at most $j+1$ members of $P_{j}$.

Where $0 \leqslant j<\{n / 2\}$,

$$
(j+1) /(n-j) \leqslant 1
$$

and therefore combining (7) and (8) we have (6), and the proof is complete.

(Paper 71B1-193) 